\title{
VIII.2 Limitationen
}

Zusammenfassend muss in der vorliegenden Studie eine Reihe von Limitation im Sinne einer selbstkritischen Betrachtung genannt werden. Bezugnehmend auf die theoretische Aufarbeitung des Themas ist deutlich festzuhalten, dass es sich vor allem bei dem Thema «Öffentlichkeit» um ein wesentliches Konzept kommunikationswissenschaftlicher (oder auch soziologischer) Forschung handelt. Es soll daher nicht der Anspruch erhoben werden, dass die theoretische Aufarbeitung abschliessend oder vollumfänglich ist. Vielmehr wurde eine Auswahl unterschiedlicher Theorie-Perspektiven getroffen, die für die Abzeichnung des Öffentlichkeitswandels relevant erscheinen. Auch die Ausführungen hinsichtlich einer digitalen Transformation der Öffentlichkeit könnten - selbstredend - durch viele weitere aktuelle Studien ergänzt werden, die schliesslich laufend weiterentwickelt werden. Zudem war es nicht das Ziel, eine neue Öffentlichkeitstheorie zu konstruieren. Wesentlich herausgestrichen wird allerdings die Annahme, dass das Phänomen der Gegenöffentlichkeit eine zentrale Rolle in aktuellen Öffentlichkeitskonzepten einnehmen sollte, da der digitale Wandel vor allem für jene Gruppen den Kommunikationsraum und die Vernetzungsmöglichkeiten massgeblich geöffnet hat. Auch hinsichtlich des Gegenöffentlichkeitsbegriffes könnten noch weitere Perspektiven miteinbezogen werden, so z. B. eine stärkere historische Einordnung, auch bezugnehmend auf das Untersuchungsphänomen der alternativen Nachrichtenmedien. Der Fokus auf die Themen «Desinformation» und «Verschwörung» ist in erster Linie der aktuellen Konnotation mit dem Alternativmedienbegriff geschuldet. Auch in diesen Bereichen ändert sich der aktuelle Stand der Forschung rasant, weswegen nur ein Überblick über die aktuelle Forschung gegeben werden konnte. Weiter nimmt das theoretische Kapitel bezugnehmend auf die relationale Analyse zwischen Öffentlichkeit und Gegenöffentlichkeit eine Sonderstellung dahingehend ein, dass es eher als methodische Begründung der vorliegenden Studie zu betrachten ist. Das Ziel bestand vordergründig darin, aufzuzeigen, inwiefern Netzwerkrelationen theoretisch erklärt werden können. Die Auswahl bestimmter Theorien ist auch in diesem Zusammenhang nicht abschliessend, sondern könnte durch zahlreiche weitere Schriften ergänzt werden.

Vor allem im Hinblick auf die empirisch-methodischen Schritte dieser Arbeit müssen wesentliche Limitationen aufgezählt werden. Das Forschungsprojekt erstreckte sich über mehrere Jahre. Entsprechend werden auch Mängel in der Konzeption weitergetragen, zumal dieses Projekt bewusst darum bemüht war, eine enge Verzahnung der einzelnen methodischen Schritte zu gewährleisten. Das primäre Forschungsinteresse entstand aufgrund der Frage, wie Alternativmedien im DACH-Raum überhaupt definiert werden können. Darauf aufbauend wurde versucht, ein möglichst breites Sample alternativer Newswebsites zu erheben. In den letzten Jahren durfte ich mit vielen Kolleg_innen über die Problematik des 
Samplings ebensolcher Seiten diskutieren - den idealen Zugang scheint dabei noch niemand gefunden zu haben. Kritisch zu hinterfragen ist erstens die Auswahl der Suchbegriffe. Diese beruhte auf bereits gesichteter Literatur zum Thema, aber auch auf empirischer Grundlage, wonach bereits gesampelte Websites nach möglichen Definitionskriterien untersucht wurden. Aufgrund des Fokusses der aktuellen Forschung wie auch der deutlichen Präsenz rechtspopulistisch gesinnter Medien, die das Motiv der Aufdeckung von «Fake News des Mainstreams» verfolgen, muss kritisch reflektiert werden, ob die gewählten Suchbegriffe das Sample nicht vielleicht stark beeinflusst oder in eine bestimmte Richtung gelenkt haben. Insofern erwies es sich auch als schwierig, alternative Nachrichtenmedien, die eine eher linkspolitische Ausrichtung auszeichnet (vorwiegend Alternativmedien der Typen III und IV) zu identifizieren. Fraglich ist, ob diese Typen aus quantitativer Sicht tatsächlich in digitalen Öffentlichkeiten unterrepräsentiert sind, oder ob dies auf einer Einschränkung des methodischen Zugriffs fusst. Für zukünftige Studien wäre es demnach essentiell, Suchbegriffe zu ergänzen - z. B. basierend auf den inhaltlichen Beschreibungen der unterschiedlichen Typen, die erst im Rahmen der Datenauswertung resultierten. Weiter kann das Sampling über die Google-Suchmaschine kritisiert werden, da die Treffer-Listen - entsprechend der Plattformlogiken - auf algorithmischer Selektion beruhen. Diese Limitation wurde zu überbrücken versucht, indem die Websites selbst auf weitere Verlinkungen auf Websites alternativer Nachrichtenmedien untersucht wurden. Auch hätte die Erhebung einschlägiger Newssites über Accounts auf Digitalplattformen erfolgen können - zumal in weiterer Folge konkret Twitter als empirischer Ausschnitt fungierte. Trotzdem wird der Zugriff über Websites als wesentlich erachtet, da dadurch ein breiteres Sample erzielt werden konnte. Die deskriptive Analyse der Zugriffszahlen hat ergeben, dass zwar viele Websites auf den Plattformen vertreten sind, dass diese allerdings häufig - vor allem Seiten mit geringeren Zugriffzahlen - ausschliesslich über deren Websites kommunizieren. Das Sample könnte in zukünftigen Studien allerdings ergänzt werden, indem beispielsweise die auf Twitter erhobenen Datensätze auf weitere einschlägige Newswebsites untersucht würden. Hinsichtlich der Kriterien, welche Websites überhaupt Teil des Samples werden, könne kritisiert werden, dass Blogs von Einzelakteur_innen ausgeschlossen wurden, sofern sie nicht als Nachrichtenseiten auftreten. Es zeigte sich, dass die Grenzen in diesem Zusammenhang fliessend sind, folglich könnte argumentiert werden, dass beispielsweise auch die reichweitenstarken Newssites Tichys Einblick oder KenFM unter dieses Kriterium fallen würden, da sie deutlich auf die Herausgeber, Roland Tichy und Ken Jebsen, der Seiten rekurrieren. Aufgrund ihrer regelmässigen Aufarbeitung aktueller Nachrichten war es aber relevant, diese in das Sample mitaufzunehmen. Prinzipiell wurden Seiten eher im Sample behalten als ausgeschlossen. Für die Erschliessung des Samples musste zudem das sehr breit gefasste Kriterium erfüllt werden, dass 
sich die Nachrichtenseiten per Selbstdefinition als Alternative zur hegemonialen Öffentlichkeit positionieren. Ziel war es, die Definition alternativer Nachrichtenmedien erst empirisch herauszuarbeiten, weswegen dieses Kriterium eher vage gefasst werden musste. Schlussendlich musste im Zuge einer ersten Analyse aller gesammelten Websites fallweise entschieden werden, ob deren Selbstverständnis in dieser Weise interpretiert werden kann. Da das Sample die Basis für die Datenerhebung auf Twitter darstellte, wurde das Sampling kurz vor dem Erhebungszeitraum auf Twitter abgeschlossen, weswegen nicht der Anspruch erhoben wird, dass es sich um eine Vollerhebung alternativer Nachrichtenmedien im DACH-Raum handelt. Vielmehr konnte schon während des Erhebungszeitraums festgestellt werden, dass Nachrichtenseiten teilweise in der Zwischenzeit gelöscht wurden; gleichzeitig konnten neue einschlägige Websites identifiziert werden, die allerdings nicht mehr Teil der Analyse waren. Dies spricht wiederum für die Annahme einer Longtail-Öffentlichkeit, wonach sich neue Kommunikator_innen auf einfache Weise Zugang zur Öffentlichkeit verschaffen können, deren Präsenz aber durchaus volatil sein kann. Abgesehen vom Sample alternativer Nachrichtenmedien mussten weitere Samples professioneller Nachrichtenmedien aus Deutschland, Österreich und der deutschsprachigen Schweiz induktiv erstellt werden. Auch hier wird nicht der Anspruch einer Vollerhebung erhoben, bzw. hätten in diesem Kontext die Erhebungskriterien allenfalls exakter eingeschränkt werden müssen.

Bezugnehmend auf die typologische Einordnung erwies sich der Pool alternativer Nachrichtenmedien als ausreichend grosse Datenbasis. Die Analyse selbst und die Erstellung unterschiedlicher Typen sind von etwaigen Mängeln in der Erhebung selbst geprägt. Nicht ausgeschlossen werden darf, dass noch weitere Typen alternativer Nachrichtenmedien existieren. So zeigte sich beispielsweise im Rahmen der Netzwerkanalysen, dass linkspositionierte Media-Watchdogs einen wesentlichen Stellenwert in der Twittersphäre einnehmen. Diese wurden dem Typ IV zugeordnet, obwohl sie tendenziell eine Sonderrolle innehaben, indem sie weniger auf die Aufbereitung und Verbreitung von aktuellen Nachrichten abzielen, sondern vielmehr eine Meta-Medienkritik vornehmen. Festzuhalten ist zudem, dass eine jede typologische Unterscheidung idealtypischen Charakter besitzt. Es ist deshalb anzunehmen, dass auch innerhalb der Typen Unterschiede bezugnehmend auf die aufgestellten Kategorien (z. B. die ökonomischen Strukturen) vorhanden sind und ausserdem Mischtypen existieren. So war beispielsweise eine Abgrenzung zwischen Typ I und Typ II nicht immer eindeutig. Auch hätte die typologische Einordnung mithilfe weiterer Daten ergänzt werden können. Für eine vollumfängliche Analyse der Medieninhalte wäre es für zukünftige Studien notwendig, Medienbeiträge (quantitativ oder qualitativ) inhaltsanalytisch auszuwerten. Auch könnten weitere Aspekte, wie beispielsweise eine genauere Erforschung der Finanzierung oder der politischen Abhängigkeit einzelner Nachrichtenme- 
dien, zusätzlich untersucht werden. Da sich diese Studie auf das Selbstverständnis alternativer Nachrichtenmedien aus einer phänomenologischen Perspektive fokussierte, wurde davon Abstand genommen. Vor diesem Hintergrund wurde zudem nach bestem Wissen versucht, normative Bewertungen zu vermeiden, was mich als Forscherin in vielen Fällen herausforderte. Kritisiert werden könnte beispielsweise, dass Typ II den Titel «Verschwörung und Spiritualität» trägt und Typ IV als «seriös» bezeichnet wird. Dahingehend ist indes festzuhalten, dass der Begriff «Verschwörung» in diesem Zusammenhang nicht als Kampfbegriff zu verstehen ist, sondern auf von den Medien selbst angenommene Elitenverschwörungen Bezug nimmt. Die Beschreibung als «seriös» bezugnehmend auf Typ IV verweist weiter auf das Selbstverständnis einschlägiger Medien, die sich auf traditionelle journalistische Werte berufen. Fraglich ist zudem, ob sich das jeweilige Selbstverständnis auch in der Berichterstattung selbst widerspiegelt. Zukünftige Studien könnten an dieser Stelle mit medieninhaltsanalytischen Verfahren ansetzen.

Die Erhebung der Twitterdaten erwies sich vor allem aufgrund der zu erhebenden Datenfülle als herausfordernd. Die Erstellung zweier Datensätze war jedoch essenziell für weitere methodische Schritte. So bezog sich der erste Datensatz auf die Daten der Streams alternativer Newswebsites, ausserdem wurden die Screennames der Accounts alternativer Medien miterhoben. Letztere wurden zusätzlich erfasst, um ein möglichst umfangreiches Bild von Alternativmedien in der deutschsprachigen Twittersphäre zu generieren. Für die Streams professioneller Nachrichtenmedien aus dem DACH-Raum war dieses Vorgehen jedoch nicht möglich, da die Anzahl an Keywords überschritten worden wäre. Deswegen basieren die Netzwerke alternativer und professioneller Nachrichtenmedien nur auf den URLs dieser und nicht - wie im Fall des Datensatzes der Alternativmedien - auch auf den Screennames. Dennoch ist davon auszugehen, dass die Ergebnisse dadurch nicht verzerrt werden, da für den Forschungsgegenstand vor allem die Einbettung von URLs in der Twittersphäre massgeblich erscheint. Einhergehend mit der hohen Anzahl an Suchbegriffen in den Twitter-Streams war es zudem wesentlich, die Datensätze gründlich zu bereinigen. Ansonsten wären viele «false positives» Teil der Grundgesamtheit gewesen. Grund hierfür ist, dass die Keyword-Suche auch Fälle miterhebt, in denen Teile der URLs in einem anderen Kontext in Tweets implementiert sind (z. B. tt.com als URL der Tiroler Tageszeitung, was aufgrund der Kürze des URLs viele weitere Texte in der Twittersphäre für den Forschungskontext fälschlicherweise miterhebt). Vor allem in den deutschen und österreichischen Streams wurde daher eine Vielzahl an Daten erhoben, die erst aus den Datensätzen gelöscht werden mussten. Durch die Fülle an Daten, die erhoben wurden, kam es auch teilweise zu Unterbrechungen der Streams. Diese wurden zwar schnellstmöglich behoben, dennoch resultierten daraus fehlende Daten. Dies hätte vermieden werden können, indem die Keywords (in Form von 
URLs) vor der Datenerhebung intensiver bereinigt worden wären. Aus zeitlichen Gründen und um grössere Datenausfälle im Erhebungszeitraum zu verhindern, war dies allerdings nicht mehr möglich. Der Datensatz alternativer Medien blieb von etwaigen Datenausfällen allerdings zum grössten Teil verschont. Auch im Zuge der Erhebung der Follower_innen für die Netzwerkanalysen konnten nicht für jeden identifizierten Account Daten erhoben werden - beispielsweise, weil Accounts in der Zwischenzeit gelöscht oder gesperrt wurden, obwohl die Erhebung der Follower_innen möglichst zeitnah erfolgte. Für zukünftige Studien könnten zudem die erhobenen Datensätze aus einer quantitativen Perspektive stärker analysiert werden, so auch die in dieser Arbeit deskriptiven Beschreibungen der Netzwerkstrukturen. Da in dieser Arbeit der Fokus auf qualitativen Interpretationen aus einem phänomenologischen Blickwinkel lag, wurde quantitativen Analysen weniger Bedeutung zugemessen, obgleich diese weitere fruchtbare Erkenntnisse liefern könnten.

Ausserdem kann hinterfragt werden, ob Twitter der geeignete empirische Ausschnitt der Netzwerkanalysen ist. So wäre beispielsweise auch ein Vergleich mit Facebook oder YouTube interessant, insbesondere im Hinblick auf eine durch den digitalen Wandel evozierte Plattformisierung der öffentlichen Kommunikation. Twitter stellt demnach nur einen Ausschnitt der digitalen Öffentlichkeit dar, obgleich die Plattform vor allem für die Analyse von Nachrichtenmedien einen geeigneten Untersuchungsausschnitt darstellt.

Schliesslich liess sich im Rahmen der interpretativen, qualitativen Analyse der Netzwerkstrukturen deutlich erkennen, dass eine Herausforderung qualitativer Forschung darin besteht, geeignete Samples aus der Datenfülle zu ziehen, die aus computergestützten Verfahren resultiert. Da in diesem Zusammenhang kaum Best-Practice-Beispiele aus bestehenden Forschungen existieren, wurden selbständig Kriterien für die Auswahl «geeigneter» Tweets definiert, die qualitativ inhaltsanalytisch untersucht wurden. Auch hier stellt sich die generelle Frage, wo die Grenzen der qualitativen Datenselektion gezogen werden. Ich gehe jedoch davon aus, dass die Auswahl geeignet war, um die zugrundeliegende Forschungsfrage zu beantworten. Ein Mehr an Daten steht - zumindest im Rahmen qualitativer Analysen - nicht gleichbedeutend für ein Mehr an Erkenntnis.

\section{VIII.3 Ausblick}

Diese Arbeit bemühte sich darum, aus einer phänomenologischen Perspektive Alternativmedien im deutschsprachigen Raum zu untersuchen. Ich möchte mir abschliessend dennoch einen normativen Blick auf den Untersuchungsgegenstand erlauben. Alternative Medien nehmen prinzipiell - wenn sich auf das Definitionskriterium der Vierten Gewalt berufen wird - eine zentrale Rolle in demokratischen 